\title{
Research on Leakage Detection and Analysis of Leakage Point in the Gas Pipeline System
}

\author{
Zhao Yang ${ }^{1 *}$, Mingliang Liu, Min Shao, Yingjie Ji \\ Department of Thermal Engineering, Tianjin University, Tianjin, China \\ E-mail: "zhaoyang@tju.edu.cn \\ Received June 23, 2011; September 2, 2011; accepted September 15, 2011
}

\begin{abstract}
Recently, with large-scale use of natural gas and massive constructions of gas pipelines, more and more public concern is focused on pipeline leakage. The leakage caused by holes on gas pipelines generates economic losses to gas companies and causes risks to the environment and sometimes accidents. In order to detect and locate pipeline rupture immediately, the leakage detection method plays a key role in the overall integrity management in the pipeline system. One of the most important applications of transient simulation is dynamic leakage detection. A leakage detection model and the solution were proposed based on the three conservation laws in hydromechanics and the state equation, which includes transient simulation model and volume balance model. Dynamic parameters involved in the model such as pressure, flow and temperature can be acquired through SCADA (Supervisory Control and Data Acquisition) system. By analyzing the factors influencing leakage position, we came to a conclusion that leakage and outlet pressure are more important parameters compared to the coefficient of frictional resistance and pipeline diameter. The more leakage increases, the closer leakage point approaches pipeline outlet. Leakage location is closer to outlet when pipeline outlet pressure becomes bigger. Experiments were also carried out according to leakage percentage.
\end{abstract}

Keywords: Leakage Detection, Transient Simulation, Leakage Location, SCADA

\section{Introduction}

Natural gas is becoming an important energy resource in China because of its cleanness and high unit-calorie. Pipeline transportation is one of the most efficient ways to convey natural gas. Pipeline rupture make sudden change in pressure, causing economic and environmental problems without detecting the leakage position and repairing in time [1]. When meeting an ignition source, the leaked gas may lead to a flame jet, even form a horrible explosion under proper conditions. The bow-waves of explosion can kill many lives and destroy buildings. The traditional way to avoid tragedy is perambulation by workers. Since the efficiency of perambulation by workers is pretty low, the leakage detection of gas pipeline with online software plays a key role in the overall integrity management of the pipeline system [2].

With the development of computer technologies and SCADA (Supervisory Control And Data Acquisition) system of gas pipeline, various leakage detection methods based on software are proposed and improved constantly. Pipeline leak detection technologies basically are divided into two categories: online leak detection system and discrete leak detection system [3]. According to API 1130, conventional pipeline leak detection systems should be based on computational pipeline monitoring (CPU) [4]. A number of pipeline leak detection models have been implemented on several pipeline systems [5]. Leakage detection technologies include the following methods [6], the volume-mass balance method, the pressure monitoring method with statistical analysis and/or pattern matching, acoustic monitoring method, the transient leakage detection method, etc. However, many methods are hedged in with their shortcomings, which are, long response time, and incidence of false alarm reporting, etc. [7].

The transient leakage detection is used to monitor whether pipeline is in a normal state by establishing the accurate pipeline model and utilizing perfect numerical methods. Comparatively speaking, the transient leakage detection method has the advantages of speediness and exactness. Gas pipeline leakage detection research based on transient simulation had begun since the early 1980s abroad. The transient leakage detection is a major appli- 
cation of transient simulation software, aiming to detect and locate pipeline rupture immediately. In Japan, gas pipeline leakage detection technology using the online simulation method has been used on Niigata-Sendai pipeline in 2000. On account of the SCADA system of gas pipeline hasn't been developed adequately in China, the technique was just considered to be primary. In this paper, the factors influencing leakage position were analyzed.

\section{General Description of the Model}

\subsection{Mathematic Model Base}

The flow of gas in pipes can be divided into two situations. One is that no heat is exchanged between gas in pipeline and the soil, the other is that the heat is totally transferred, which means the temperature of gas in pipeline is the same as the soil's [8]. In order to describe the model conveniently, some assumptions are presented as follows [9]: 1) The gas flow in pipeline can be described as a one-dimensional approach, which means the parameters are considered to be identical on a section. 2) The effect of natural gas on external environment is negligible, that is to say, the soil temperature is constant. 3) The gas temperature equals to the temperature of pipeline's inside wall. 4) The heat transfer process between the soil and the gas in pipes is steady.

\subsection{Transient Simulation Model}

As the transient simulation is a kind of numerical method, accurate equations are had to established to describe the model. The model is based on solutions of the system of partial differential equations that describe the conservation of mass, momentum and energy. State equation is compulsory. The equations are presented as follows:

Continuity Equation:

$$
\frac{\partial \rho}{\partial \tau}+\frac{\partial(m)}{\partial x}=0
$$

Momentum Equation:

$$
\frac{\partial(m)}{\partial \tau}+\frac{\partial}{\partial x}\left(\rho+\frac{m^{2}}{\rho}\right)=-g \rho \frac{\mathrm{d} s}{\mathrm{~d} x}-\frac{\lambda}{2} \frac{v^{2}}{d} \rho
$$

Energy Equation:

$$
\begin{aligned}
& \frac{\partial}{\partial \tau}\left[\left(h-\frac{p}{\rho}+\frac{m^{2}}{2 \rho^{2}}\right) \rho\right] \\
& +\frac{\partial}{\partial x}\left[\left(h+\frac{m^{2}}{2 \rho^{2}}\right) m+\frac{4 k\left(T-T_{0}\right)}{d}+m g \frac{\mathrm{d} s}{\mathrm{~d} x}\right]=0
\end{aligned}
$$

State equation:

$$
\frac{p}{\rho}=Z R T
$$

Partial differential equations are converted to ordinary differential equations with method of characteristics as shown in Figure 1.

Because Equations (1)-(3) are conservation equations, the following formula (5) can substitute for them.

$$
\begin{gathered}
\frac{\partial A}{\partial t}+\frac{\partial B}{\partial x}=C \\
\frac{\partial A}{\partial t}=\frac{\Delta A}{\Delta t}=\frac{A_{i}^{k+1}-A_{i}^{k}+A_{i+1}^{k+1}-A_{i+1}^{k}}{2 \Delta t} \\
\frac{\partial B}{\partial x}=\frac{\Delta B}{\Delta x}=\frac{B_{i}^{k+1}-B_{i}^{k}+B_{i+1}^{k+1}-B_{i+1}^{k}}{2 \Delta x} \\
C=\frac{C_{i}^{k+1}+C_{i}^{k}+C_{i+1}^{k+1}+C_{i+1}^{k}}{4}
\end{gathered}
$$

\subsection{Volume-Mass Balance Model}

In a period of time, the fluid volume loss due to leakage is equal to the sum of the inlet/outlet volume difference value and the change in fluid inventory of the pipeline. The change in fluid inventory of the pipeline influenced by temperature and pressure include two parts: the variation of steel pipeline volume and the fluid volume.

The Volume-mass balance equations are presented as following:

$$
\begin{gathered}
\Delta V=V_{\text {in }}-V_{\text {out }}-\Delta I \\
\Delta I=\Delta I_{\text {st }}+\Delta I_{F} \\
\Delta I_{s t}=V_{0}\left[\frac{\Delta P_{a v g} \cdot D}{e \cdot E}\left(1-\mu^{2}\right)+\alpha \cdot \Delta T_{a v g}\right] \\
\Delta I_{F}=V_{0}\left[\frac{\Delta P_{a v g}}{K}-\beta \cdot \Delta T_{\text {avg }}\right]
\end{gathered}
$$

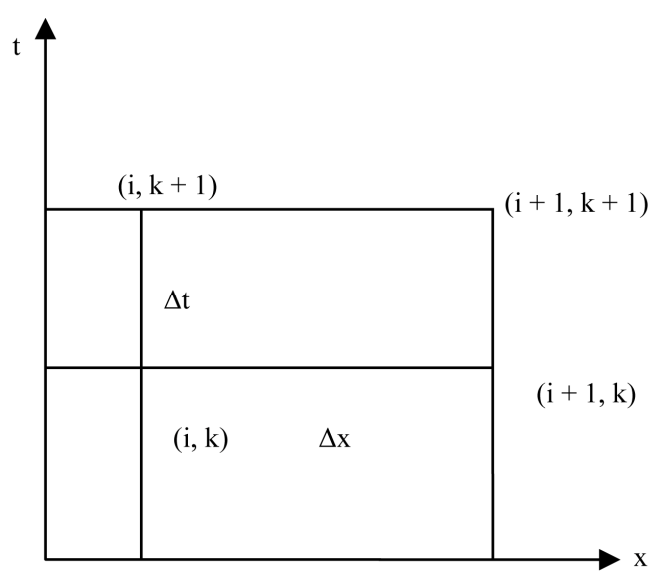

Figure 1. Solving of the transient simulation model. 
Two independent variables of pressure and temperature can be acquired on the basis of the volume balance and the transient simulation, collected through SCADA. Then average pressure and average temperature in a period of time are calculated, which are substituted into Equations (11) and (12) to get the variation of steel pipeline volume $\Delta I_{s}$ caused by temperature and pressure and volume change of fluid $\Delta I_{F}$ caused by density. After substituting $\Delta I_{s}$ and $\Delta I_{F}$ into Equation (8), fluid inventory of the pipeline $\Delta I$ can be counted. Finally, by means of Equation (9), leakage volume will be acquired.

\subsection{Leakage Detection System}

The leakage detection model includes transient simulation model and volume-mass balance model. When leakage happens, leakage location can be calculated by the following formulas according to the relation between flow and pressure, which gas flow in pipeline is in a steady state [10].

$$
\begin{gathered}
P_{D}-P_{X}=\frac{f_{1} \rho_{1} X Q_{D}^{2}}{2 A^{2}}+\rho_{1} g\left(h_{X}-h_{D}\right) \\
P_{X}-P_{S}=\frac{f_{2} \rho_{2}(L-X) Q_{S}^{2}}{2 A^{2}}+\rho_{2} g\left(h_{S}-h_{X}\right)
\end{gathered}
$$

In Equations (13) and (14), subscripts $D$ and $S$ denote outlet and inlet of the pipeline separately, while $X$ represents leakage location. The parameter $h$, which stands for elevation, is really worthy to be discussed. In the highpressure transmission and distribution system, the pressure drop due to elevation changes is usually smaller than that caused by friction by $5 \%$. So, elevation corrections are considered only when the elevation diversification exceeds 100 meters every kilometer in the highpressure transmission and distribution system. Sometimes, we must add elevation correction in flow calculation equations [11].

The implemented leakage detection system is illustrated in Figure 2. The system contains five modules:

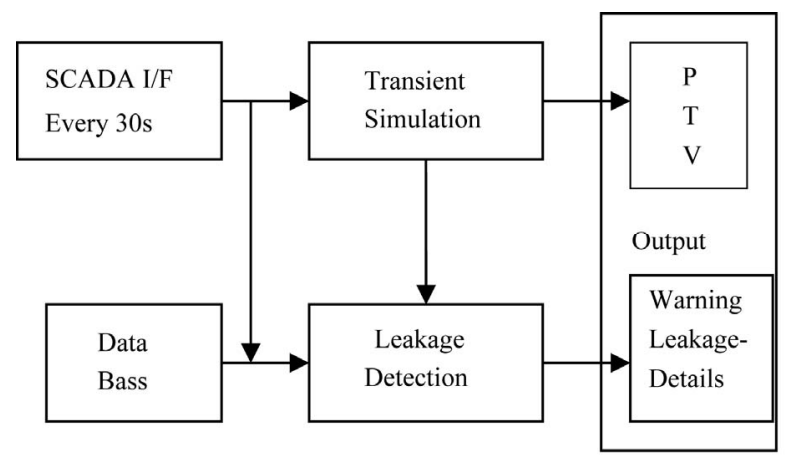

Figure 2. Leakage detection system.
SCADA I/F, data Bass, Transient Simulation, Leakage Detection, Output.

Our method can make full use of actual data by SCADA (Supervisory Control And Data Acquisition) system. It means the accuracy is assured. The weakness is a mass of sensors are required. Some other leakage detection methods contain acoustic emission method [12] and wavelet transform theory [13]. Several weaknesses are obvious. Acoustic emission method has the limitation of distance of the sound. The accuracy of wavelet transform theory is up to the instrument measurement precision.

\subsection{SCADA I/F Model}

The SCADA system has the function of transferring the acquired data from a pipeline system to Transient Simulation Model every 30 seconds. This module communicates with SCADA. Dynamic parameters are collected every 30 seconds, such as pressure, flow and temperature.

\subsection{Transient Simulation Model}

Transient flow is simulated utilizing perfect numerical methods based on actual data. Pressure and temperature served as independent variables are provided in order to get average pressure and average temperature. We can switch the Equations (13) and (14) to different equations which can be solved with boundary information by virtue of finite difference method. Then all the parameters of the gas in the pipeline system can be acquired.

\subsection{Leakage Detection}

The leakage detection is carried out by comparing the data acquired through the SCADA system with that by the Transient Simulation Model. This model could provide leakage point judgment and prompt warning based on transient simulation and volume balance.

\subsection{Output}

To get a supervisory control of the parameters and give an alarm when something abnormal happens, some outputs are necessary. This model includes leakage details, warning message and parameter values, which includes pressure, flow, temperature, density, and so on.

\section{Factors Influencing Leakage Location}

\subsection{Leakage Amount and Leakage Location}

Suppose pipeline inlet pressure and outlet pressure are evaluated 1,650,000 $\mathrm{Pa}$ and 1,600,000 $\mathrm{Pa}$ separately. Inlet 
flux is initially set at $3 \mathrm{~m}^{3} / \mathrm{s}$. Leakage percentage ranges from $0.3 \%$ to $93 \%$ of the nominal gas flow. Curves on Figure 3(a) describes the relation between leakage position and leakage amount when pipe diameters varies from $400 \mathrm{~mm}$ to $700 \mathrm{~mm}$, while Figure 3(b) shows us that how the coefficient of frictional resistance influences leakage position. Obviously, the smaller the pipe diameter is, the further the leakage point is apart from pipeline inlet under the same other conditions. The trend is similar to the relationship between the coefficient of frictional resistance and leakage point. However, the curves based on different pipe diameters and the coefficient of frictional resistance are so close to each other, which means these two parameters influence leakage position pretty slightly. Compared to pipe diameter and the coefficient of frictional resistance, Figures 3(a) and (b) show that the leakage amount is a more important parameter.

When outlet flux is larger than $2.8 \mathrm{~m}^{3} / \mathrm{s}$, it could hardly detect whether leakage happens and locate leakage position, since even if gas flow down pipeline is in a normal state, flow loss may be created due to friction.

The more leakage amount increases, the closer leakage position approaches pipeline outlet. When leakage percentage ranges from $0.3 \%$ to $33 \%$ of the nominal gas

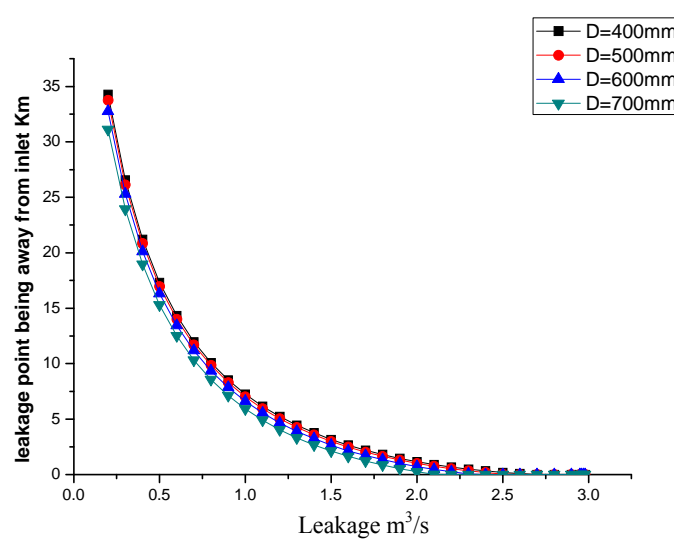

(a)

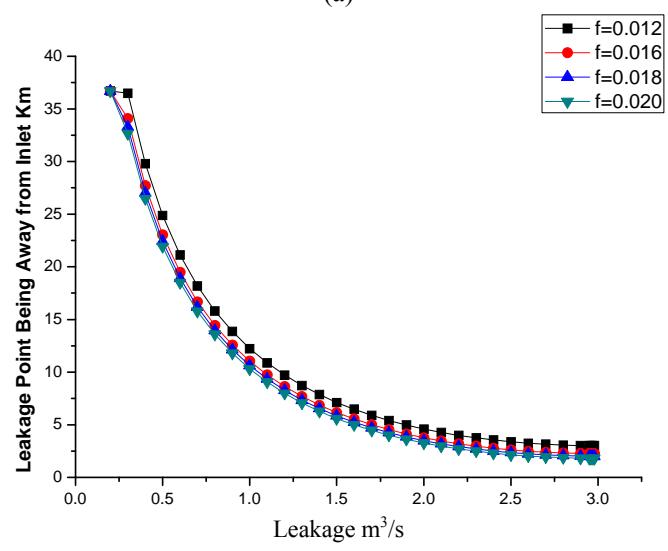

(b)

Figure 3. Relation between leakage point and leakage. flow, leakage location is moving from 36.7 kilometers to less than 10 kilometers away from pipeline inlet. However, with the leakage percentage continues growing, leakage location has little diversification. The leakage point is always less than 10 kilometers apart from pipeline inlet. The reason for the trend of the curve in Figure 4 will be elaborated in next section.

For pipeline DX section, it satisfies the equation $\frac{P_{D}^{2}-P_{X}^{2}}{x}=k Q^{2}$, while for pipeline XS section, it meets the equation $\frac{P_{X}^{2}-P_{S}^{2}}{L-X}=k Q^{2}$. Therefore, for the same pipeline, any point pressure can be expressed as Equation (15).

$$
P_{X}=\sqrt{P_{D}^{2}-\left(P_{D}^{2}-P_{S}^{2}\right) \frac{X}{L}}
$$

According to Equation (10) and Figure 4, it is clearly shows that the further the point is apart from pipeline inlet, the faster pressure descends. The pressure of the whole pipeline descends by half in 3/4 length of pipeline away from inlet [14]. When leakage point is closer to inlet, the pressure is higher, so differential pressure between gas and atmosphere is still bigger, and leakage is even more. It means differential pressure is even bigger and leakage location is nearer to pipeline inlet when leakage is much more.

\subsection{Outlet Pressure and Leakage Location}

Suppose that pipeline inlet pressure is initially set at $1,650,000 \mathrm{~Pa}$ and inlet flux $3 \mathrm{~m}^{3} / \mathrm{s}$. The outlet pressure ranges from $1,650,000 \mathrm{~Pa}$ to $100,000 \mathrm{~Pa}$ to guarantee it higher than atmospheric pressure. The relation between leakage location and pipeline outlet pressure has been studied, which is described in Figure 4.

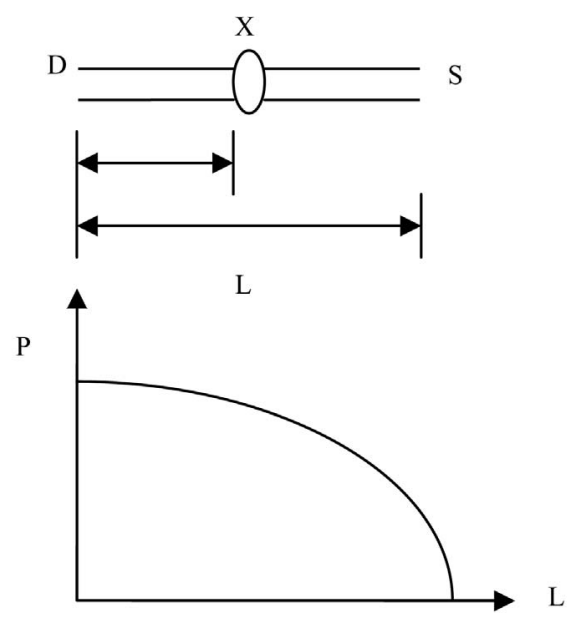

Figure 4. Gas pipeline and pressure changes. 
Referring to Figure 4, it is apparent that outlet pressure and leakage position almost reveal the linear relation. When pipeline outlet pressure constantly goes on, the distance of leakage point apart from inlet becomes further. With outlet pressure perpetually ascending, differential pressure between pipeline gas and atmosphere keeps on increasing. For the reason of that, leakage location is closer to outlet when pipeline outlet pressure becomes bigger under the same other conditions.

\section{Conclusions}

The present work clearly shows the advantages of an online computing technique for pipeline supervision [15]. The computational method which permits to detect and locate leakage is based on the on-line analysis of signals originated from pressure, flow and temperature acquired by SCADA.

Leakage detection model is set up based on continuity equation, momentum equation, energy equation, state equation and volume-mass balance. THE leakage detection model includes five modules: SCADA I/F, Dada Bass, Transient Simulation, Leakage Detection, Output. Leaks as small as $0.3 \%$ of the nominal gas flow are readily detected. When leakage point is much closer to inlet, the pressure is even higher, so differential pressure between gas and atmosphere is still bigger, and leakage is even more. The pipeline outlet pressure and leakage position almost reveal the linear relation. The results show that leakage and outlet pressure are more important parameters compared to the coefficient of frictional resistance and pipeline diameter. A computer program to run on-line has been developed to obtain leakage location and performs well when leakage percentage ranges from $0.3 \%$ to $93 \%$ of the nominal gas flow.

So the developed program software turns out to be a very useful tool in automatic supervision of pipelines as well as instantaneous leakage detection.

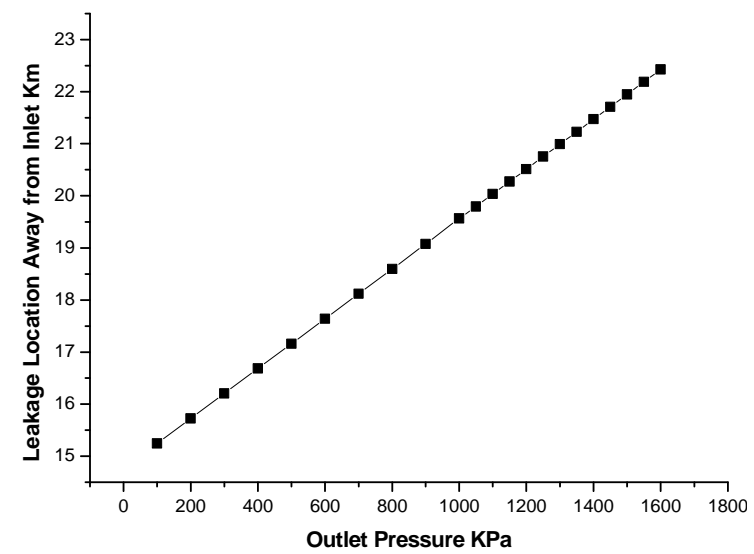

Figure 5. Relation between leakage locations and outlet pressure.
Although the transient simulation method has advantages such as rapidity and convenience, the precision of the leakage location is still a problem. An approximate range where the leakage happens can be found, but not the exact point. Another unavoidable problem is that it can hardly detect the leakage position when leakage percentage is less than $0.3 \%$ of the nominal gas flow.

To make our project perfect, we need to do some more meaningful research on the following difficulties: 1) Dispel frequent false alarms when there is no leak in the pipeline, 2) Reduce the response time 3) Increase the accuracy of leakage location.

\section{Acknowledgements}

This project is supported by the Hi-tech Research and Development Program of China (2007AA05Z200), and by NSFC, National Education Department for Doctor Center Foundation (200800560041), Science and Technology Sustaining Project of Tianjin City (07ZCGYSF02600, 07ZCGYSF01500).

\section{References}

[1] C. Sandberg, J. Holmes, K. McCoy and H. Koppitsch, "The Application of a Continuous Leak Detection System to Pipelines and Associated Equipment," IEEE Transactions on Industries Application, Vol. 25, No. 5, 1989, pp. 906-909. doi:10.1109/28.41257

[2] F. Kenya, M. Reiko, K. Akira, S. Hitoshi and K. Ichiro, "Gas Pipeline Leak Detection System Using the Online Simulation Method," Computers and Chemical Engineering, Vol. 24, No. 2-7, 2000, pp. 453-456. doi:10.1016/S0098-1354(00)00442-7

[3] RELI, "A Review of Pipeline Leak Detection Methods," REL Instrumentation Project Report, Shell International Exploration and Production Limited, London, 1997.

[4] D. Scott, "API Document for Leak Detection," Oil and Gas Journal, Petroleum News, Tulsa, 1999.

[5] X. J. Zhang, "Statistical Leak Detection in Gas and Liquid Pipeline," Piepes \& Pipelines International, Vol. 38, No. 4, 1993, pp. 20-26.

[6] E. B. Liu, S. B. Peng and C. J. Li, "Discussion of Modern Pipeline Leak Detection Techniques," Pipeline Technique and Equipment, Vol. 5, 2004, pp. 17-19.

[7] K. E. Abhulimen and A. A. Susu, "Modelling Complex Pipeline Network Leak Detection Systems," Process Safety and Environmental Protection, Vol. 85, No. 6, 2004, pp. 579-598. doi:10.1205/psep06069

[8] A. J. O. Andrzej and C. Maciej, "Comparison of Isothermal and Non-Isothermal Pipeline Gas Flow Models," Chemical Engineering Journal, Vol. 81, No. 1-3, 2000, pp. 41-51.

[9] Z. Yang, "Theoretical Research and Application of 
Steady and Transient Simulation of Gas Network," $\mathrm{Na}$ tural Gas Industry Journal, Vol. 26, No. 4, 2006, pp. 105-108.

[10] X. K. Xing, “A Simulation Method of Leakage Detection in Product Pipeline," Pipeline Technique and Equipment, Vol. 3, 2000, pp. 12-14.

[11] Y. J. Li, "Design and Practice of Transmission and Distribution System," China Construction Industry Press, Beijing, 2007, pp. 152-153.

[12] B. Van Hieu, S. Choi, Y. Uk. Kim, Y. Park and T. Jeong "Wireless Transmission of Acoustic Emission Signals for Real-Time Monitoring of Leakage in Underground Pipes", KSCE Journal of Civil Engineering, Vol. 15, No.
5, 2011, pp. 805-812. doi:10.1007/s12205-011-0899-0

[13] Z. Yang, Z. Xiong and M. Shao, "A New Method of Leak Location for the Natural Gas Pipeline Based on Wavelet Analysis," Energy, Vol. 35, No. 9, 2010, pp. 38143820. doi:10.1016/j.energy.2010.05.034

[14] S. L. Wang and H. J. Zhao, "Design and Management of Gas Pipeline," Chemical Industry Press, Beijing, 2006, pp. 101-103

[15] A. S. Relnaldo, M. B. Clsudio, L. C. Sandra, J. A. F. R. Pereira, "Pressure Wave Behavior and Leak Detection in Pipeline," European Symposium on Computer Aided Process Engineering, Vol. 20, Supplement 1, 1996, pp. 491496. 


\section{APPENDIX}

Table 1. (Iterative) algorithmic (In \%) relative (absolute) efficiency/gain for $f(x)=\exp (x)$.

\begin{tabular}{cccc}
\hline Items $\downarrow$ & $\mathrm{n} \rightarrow 3$ & 6 & 9 \\
\hline PRE_PFB (f;x)[n] & 7.7098 & 7.97506 & 8.0614 \\
PRE_PDFBV (f;x)[n] & 0.0004 & 0.00000 & 0.0000 \\
PRG_PDFBV(f;x)[n] & 99.994 & 100.000 & 99.999 \\
\hline
\end{tabular}

Table 2. (Iterative) algorithmic (In \%) relative (absolute) efficiency/gain for $f(x)=\ln (2+x)$.

\begin{tabular}{cccc}
\hline Items $\downarrow$ & $\mathrm{n} \rightarrow 3$ & 6 & 9 \\
\hline PRE_PFB $(\mathrm{f} ; \mathrm{x})[\mathrm{n}]$ & $\mathbf{5 . 0 9 7 0}$ & $\mathbf{5 . 0 2 1 7}$ & 4.9961 \\
PRE_PDFBV (f;x) $[\mathrm{n}]$ & $\mathbf{0 . 0 0 0 1}$ & $\mathbf{0 . 0 0 0 0}$ & $\mathbf{0 . 0 0 0 0}$ \\
PRG_PDFBV (f;x) $[\mathrm{n}]$ & $\mathbf{9 9 . 9 9 6}$ & $\mathbf{1 0 0 . 0 0}$ & $\mathbf{9 9 . 9 9 9}$ \\
\hline
\end{tabular}

Table 3. (Iterative) algorithmic (In \%) relative (absolute) efficiency/gain for $f(x)=\sin (2+x)$.

\begin{tabular}{cccc}
\hline Items $\downarrow$ & $\mathrm{n} \rightarrow 3$ & 6 & 9 \\
\hline PRE_PFB (f;x)[n] & 5.0404 & 5.3105 & 5.4020 \\
PRE_PDFBV (f;x)[n] & 0.0004 & 0.0000 & 0.0000 \\
PRG_PDFBV (f;x)[n] & 99.991 & 99.999 & 99.999 \\
\hline
\end{tabular}

Table 4. (Iterative) algorithmic (In \%) relative (absolute) efficiency/gain for $f(x)=10^{x}$.

\begin{tabular}{cccc}
\hline Items $\downarrow$ & $\mathrm{n} \rightarrow 3$ & 6 & 9 \\
\hline PRE_PFB $(\mathrm{f} ; \mathrm{x})[\mathrm{n}]$ & $\mathbf{1 6 . 1 1 2}$ & $\mathbf{1 7 . 4 9 8}$ & $\mathbf{1 7 . 9 3 5}$ \\
PRE_PDFBV $(\mathrm{f} ; \mathrm{x})[\mathrm{n}]$ & $\mathbf{0 . 0 1 2 0}$ & $\mathbf{0 . 0 0 0 0}$ & $\mathbf{0 . 0 0 0 0}$ \\
PRG_PDFBV $(\mathrm{f} ; \mathrm{x})[\mathrm{n}]$ & $\mathbf{9 9 . 9 2 5}$ & $\mathbf{9 9 . 9 9 9}$ & $\mathbf{9 9 . 9 9 9}$ \\
\hline
\end{tabular}

